\title{
Physical and Radiobiological Evaluation of Accelerated Intensity Modulated Radiotherapy for Locally Advanced Head and Neck Cancer and Comparison with Short-Term Clinical Outcomes
}

\author{
Rekha Reddy Buchapudi ${ }^{1}$, Ravikumar Manickam ${ }^{1 *}$, Anil Kumar M R², Tanvir \\ Pasha $C ~ R^{2}$, Varatharaj Chandraraj ${ }^{1}$, Anil Pyakuryal ${ }^{3}$, Ganesh Narayanasamy ${ }^{4}$
}

\begin{abstract}
Objective: The present study aims to evaluate the accelerated intensity modulated radiotherapy (IMRT) of head and neck (HandN) treatments using physical indices and radiobiological models with its clinical correlation using histogram analysis in radiation therapy (HART). The radiobiological evaluation in terms of tumor control probability (TCP) and normal tissue complication probability (NTCP) indices were compared with acute toxicity. Materials and Methods: A total of twenty patients with stage III and IV of HandN cases treated with accelerated IMRT using 6MV photons were chosen for the study. Using HART software, physical indices of the IMRT plans have been defined by universal plan indices (UPI's) which summarize the various recognized plan indices. The overall quality factor (QF) of a plan was determined by a linear combination of all indices in UPI set. The clinical outcomes in terms of the acute toxicity like dysphagia and xerostomia were compared with NTCP values of the OAR calculated from HART software. Results: The mean QF and the mean Poisson TCP index was found to be $0.993 \pm 0.02$ and $0.86 \pm 0.02$ respectively. The mean JT Lyman NTCP index for bilateral parotid, constrictors, and larynx were found to be $0.23 \pm 0.14,0.30 \pm 0.17$ and $0.22 \pm 0.15$ respectively. The acute toxicities in terms of severity of xerostomia and dysphagia have shown a moderate correlation with NTCP values of bilateral parotids, constrictors, and larynx, respectively. Conclusion: The mean QF based on UPI was found to be close to unity, which correlates with being a better IMRT plan. The present study suggested the existence of a moderate correlation between the calculated NTCP values and their respective severities of the organ at risk (OAR's). Accelerated IMRT with chemotherapy is a clinically feasible option in the treatment of locally advanced head and neck squamous cell carcinoma (HNSCC) with encouraging initial tumor response and acceptable acute toxicities.
\end{abstract}

Keywords: Head and neck- histogram- TCP- NTCP- IMRT

Asian Pac J Cancer Prev, 20 (8), 2463-2470

\section{Introduction}

The main objective of the radiotherapy delivery is to give the maximum dose to the tumor cells and the minimum possible dose to the organ at risk (OAR). It is possible to achieve this, only when the separation between tumor control probability (TCP) and normal tissue complication probability (NTCP) plot is wide enough. However, this reduces at higher prescription doses of the tumor (Baumann et al., 2005). The advancement in imaging and modern, sophisticated techniques have increased the complexity of treatment planning while achieving the above objectives. It leads to results in maximum dose to the tumor and dose reduction to the normal structures surrounding the target (Kataria et al., 2012).

Intensity modulated radiotherapy (IMRT) is one of the modalities in which we can achieve the escalation of the target doses as well as the reduction of normal tissue complications. The dose distributions of IMRT plans are much Heterogeneous when compared to 3D conformal radiation therapy (3DCRT), which leads to a challenging process, in particular, to head and neck (HandN) cancer treatment. The main complication associated with IMRT of head and neck cases were dysphagia and xerostomia. Irradiation of swallowing structures like larynx and pharyngeal constrictors are the main reason behind dysphagia and xerostomia being noticed when the bilateral

${ }^{1}$ Department of Radiation Physics, ${ }^{2}$ Department of Radiation Oncology, Kidwai Memorial Institute of Oncology, Bangalore, India, ${ }^{3}$ Department of Physics and Engineering, University of District of Columbia, Washington DC, ${ }^{4}$ Department of Radiation Oncology, University of Arkansas for Medical Sciences, Little Rock, AR, USA. *For Correspondence: drmravi59@yahoo.com 
parotid gland is getting irradiated (Narayanasamy et al., 2015).

The dose evaluation of these kinds of IMRT plans can be visualized in the form of dose-volume histograms (DVHs). DVH is the relationship between the dose distribution of a specific organ and $100 \%$ normalized volume of such organs, and it is generated based on 3D reconstructed images in the treatment planning systems (Cadman et al., 2002). Unfortunately, the DVH contains a large volume of data, lines, and curves which tends to complicate the problem rather than making it simple for analysis. Hence, there is a need to find a simple tool to analyze the IMRT plan outcome.

The accuracy of these IMRT plans were assessed using the universal plan indices (UPI's), which takes into account the dose homogeneity, conformality, and sparing of normal tissues. As of now, there is no single parameter to assess this, and the search is still ongoing. Hence, a more practical approach of analyzing radiotherapy plans is to utilize the UPI set and the overall quality factor $(\mathrm{QF})$ of the treatment as discussed in the literature (Pyakuryal et al., 2010). The biological models such as tumor control probability (TCP) based on the Poisson statistics model and normal tissue complication probability (NTCP) based on the Lyman-Kutcher-Burman model, were efficient to estimate the radiobiological outcomes of the treatments by taking into account of the dose-volume effects in the organs. The present study aims to evaluate UPI, QF, and radiobiological outcome of IMRT for locally advanced head and neck squamous cell carcinomas using HART (Histogram Analysis in Radiation Therapy, https://hart. research.uic.edu/). HART is an open-source tool based on MATLAB (Version 7.8.0. Mathworks Inc., Natick, MA) program. It is an automated computational environment that was developed for the precise computation of dosevolume statistics for radiation therapy research. The screenshot of the graphical user interface (GUI) of the HART software is shown in Figure 1.

\section{Materials and Methods}

A total of 20 patients of stage III and IV cases of head and neck squamous cell carcinoma (HNSCC) treated with accelerated IMRT technique with concurrent chemotherapy were enrolled for this study. This study has been approved from the institutional review board, and written informed consent was obtained from each patient before enrolling in this study. Delineation of the tumor and normal tissues were done using danish head and neck cancer group (DAHANCA) contouring guidelines. The radiotherapy was delivered to a dose of 70Gy in 35 fractions to the primary and involved lymph nodes, 63Gy to intermediate-risk areas and 56Gy to lower risk areas, Monday to Saturday, six days per week with an expected overall treatment time of six weeks.

The accelerated simultaneous integrated boost (SIB) IMRT plans were optimized using Eclipse treatment planning systems (TPS) (Version 8.1, Varian Medical Systems, USA), and treated by Clinac DHX- linear accelerator using $6 \mathrm{MV}$ photons (Varian Medical Systems, USA). A radiotherapy treatment plan needs to be optimized to achieve the goal of cancer treatment. The technique of evaluating the UPI's and QF of the radiotherapy treatment plans are the most practical approach in making critical decisions in the treatments. The Cumulative DVH data from Eclipse TPS has been acquired, and parameters derived from it were used as input in HART software. HART computation was performed on a laptop personal computer with a processor speed of $2.10 \mathrm{GHz}, 4.00 \mathrm{~GB}$ of RAM, and Intel Core2 Duo (6th generation) CPU using MATLAB (version 7.8). It uses the advanced graphical features and simulation systems available in MATLAB. The MATLAB provides a flexible platform to set up a computational and graphical environment for other secondary software like HART. Though HART offers much functionality by processing radiation therapy oncology group (RTOG) and digital imaging and communications in medicine - Radiation Therapy (DICOM-RT) data file formats in the radiation therapy treatments, in the present study we have used only radiobiological-modeling for the outcome analysis (ROA) and UPI evaluation modules.

The TCP and NTCPs were calculated from the dosevolume histogram $(\mathrm{DVH})$ statistics using the Poisson statistics and JT Lyman models respectively, and the results were correlated with clinical outcomes in terms of acute toxicities of the patients with a mean follow-up period of 3 months. Statistical analysis was performed by using the paired, two-tailed student $\mathrm{t}$-test, and $\mathrm{P}<0.01$ was kept as a threshold for the significance level.

\section{(i) Evaluation of the UPIs}

In order to maintain the precision and quality of IMRT plans for HandN patients, it is mandatory to do contouring of Planning Target Volumes (PTV) and other vital organ volumes, patient immobilization, and treatment delivery utilizing an optimal treatment plan. As mentioned earlier, a single tool or parameter to quantify the quality of an IMRT plan has been ongoing but, as of now, unsuccessful. In this perspective, a simpler method of Treatment Plan Indices (TPI) evaluation technique of radiotherapy treatment has been incorporated into HART. The evaluation of the IMRT plans has been defined by UPI's, which summarize the various recognized plan indices.

The overall QF of a plan can also be determined by a linear combination of all plan indices in the UPI set. These indices can be assessed by utilizing the DVH statistics extracted from the HART. The QF can be efficiently computed for a plan by assigning the relative weights to all UPI plan indices as a complete plan evaluation strategy. Plan indices in UPI set's are systematically described as below:

\section{a) Target Coverage Index (TCI)}

The TCI accounts for the exact coverage of PTV in a treatment plan at a given prescription dose which can be expressed as:

$$
\mathrm{TCI}=\frac{\mathrm{PTV}_{\mathrm{PD}}}{\mathrm{PTV}}
$$

Where PTVPD is the PTV covered by a prescribed dose (PD) (Pyakuryal et al., 2010). 


\section{b) Critical Organ Scoring Index (COSI)}

The COSI is a measure of both target coverage and critical organ overdose. It can be expressed as:

$$
\operatorname{COSI}=\left[1-\frac{\sum_{i=1}^{N} \mathrm{w}_{\mathrm{i}} \mathrm{V}\left(\mathrm{OAR} \mathrm{R}_{\mathrm{z}} \mathrm{TOL}\right.}{\mathrm{TCI}}\right],
$$

Where $\mathrm{v}(\mathrm{OAR})_{\text {T⿰о }}$ is the fractional volume of ith organ at risk (OAR) receiving more than tolerance dose (TOL), and the relative weight (wi) of a fractional volume of each organ is $1 / \mathrm{N}$. Where $\mathrm{N}$ is the no. of organs (Menhel et al., 2006).

\section{c) Radiation Conformity Index (RCI)}

The RCI gives a consistent method for quantifying the degree of conformity based on isodose surfaces and volumes which can be expressed as:

$$
\mathrm{RCI}=\frac{\mathrm{PTV}_{\mathrm{PD}}}{\mathrm{PTV}_{0.95 \mathrm{PD}}},
$$

Where PTV0.95PD is the PTV volume covered by $95 \%$ of the prescribed dose (Knöös et al., 1998).

\section{d) Prescription Isodose Target Volume conformal index (PITV)}

The PITV assesses the conformity of a treatment plan and can be expressed as:

$$
\text { PITV }=\frac{\text { PIV }}{\text { PTV }}
$$

Where PIV is the prescription isodose volume coverage for the target and normal tissues. PITV $>1$ and PITV $<1$ refers to the over treatment and under treatment regions, respec-tively (Leung at al., 1999).

\section{e) Dose Homogeneity Index (HI) and Modified HI Index (MHI)}

HI scales the "hot" spots in and around the planning target volumes (Akpati et al., 2008). It can also be expressed as:

$$
\mathrm{HI}=\frac{\mathrm{D}_{\operatorname{Max}}}{\mathrm{PD}}
$$

and modified dose homogeneity index (MHI) is defined as:

$$
\mathrm{MHI}=\frac{\mathrm{D}_{95}}{\mathrm{D}_{5}}
$$

Where $\mathrm{D}_{\text {Max }}$ is the maximum dose point in PTV. Similarly, D95 and D5 are the doses to $95 \%$ and 5\% volume of the PTV, respectively (Yoon et al., 2007).

\section{f) Conformality Index (CI) and Conformation Number $(\mathrm{CN})$}

CI measures the conformity of a treatment plan. $\mathrm{CN}$ accounts for the relative measurement of dosimetric target coverage and sparing of normal tissues in a treatment plan. (Leung et al., 2007)

$$
\mathrm{CN}=\frac{\mathrm{TCI}}{\mathrm{TR}} \text {, and } \mathrm{CI}=\frac{1}{\mathrm{TR}}
$$

Where the treatment volume ratio (TR) is defined as:

$$
\mathrm{TR}=\frac{\mathrm{PIV}}{\mathrm{PTV} P \mathrm{PD}}
$$

\section{g) Target Volume Ratio (TVR)}

The TVR is an objective measure of how well the prescription isodose line conforms to the size and shape of the planning target volume (Paddick et al., 2000).

It is simply the inverse of ratio for PITV.

$$
\mathrm{TVR}=\frac{\text { PTV }}{\text { PIV }}
$$

\section{h) Dose Gradient Index (DGI)}

It examines the steepness or shallowness of dose fall-off in the target volume (Pyakuryal et al., 2010).

It can be expressed as:

$$
\mathrm{DGI}=\frac{\mathrm{PTV}_{\mathrm{PD}}}{\mathrm{PTV}_{0.50 \mathrm{PD}}},
$$

Where PTV0.50PD is the planning target volume coverage at $50 \%$ of $\mathrm{PD}$.

\section{i) New Conformity Index (NCI)}

$\mathrm{NCI}$ and $\mathrm{HI}$ allow for the quick and simple comparison of different radio-surgical treatment plans designed within the same or diverse radiosurgical systems, such as between LINAC and Gamma Knife. This can be used for IMRT evaluation also (Collins et al., 2006). NCI can be expressed as:

$$
\mathrm{NCI}=\frac{\mathrm{PIV} * \mathrm{PTV}}{\mathrm{PTV}_{\mathrm{PD}}^{2}}
$$

Thus, Universal Plan Indices (UPI) set can be expressed as,

$$
\mathrm{UPI}=\left\{\mathrm{X}_{\mathrm{i}}\right\}
$$

Where, $\mathrm{Xi}=$ (TCI, COSI, RCI, PITV, HI, MHI, CN, TVR, DGI, NCI), for a number of $\mathrm{N}$ major plan indices $(\mathrm{N}=10)$. The number $(\mathrm{N})$ can be arbitrarily selected from the UPI set for treatment plan evaluation in HART.

j) The quality factor (QF) of a treatment plan can be analytically expressed in terms of combination of the above set of UPI indices as given below:

$$
\mathrm{QF}=\left[2.718 \exp \left(-\sum_{\mathrm{i}=1}^{\mathrm{N}} \mathrm{W}_{\mathrm{i}} \mathrm{X}_{\mathrm{i}}\right)\right],
$$

Where the values of weight factor (Wi) can be adjusted between zero to unity for all relatively weighted indices $\{\mathrm{X}\}$ for a user-defined number of indices $(\mathrm{N})$ in the UPI set. The analytical expression in the argument of the above exponential function is also termed as the UPI function. Thus, the fundamental application of QF and UPI evaluations is to compare the conformity of plans among various trials for a treatment. Typically, QF equals to unity for an ideal plan, whereas the deviation from unity refers to underdose or overdose treatments in the corresponding plan. 
ii) Biological modeling-based outcome analysis: TCP and NTCP calculation

The outcome analysis of HandN IMRT treatments were employed using TCP and NTCP biological modeling at the HART analysis software, which offers a convenient feature for plan-specific outcome analysis (POA) of the radiotherapy treatments. The TCP model was based on the Poisson statistics model (Warkentin et al., 2004) and NTCP was evaluated using the sigmoidal dose response (SDR) model based on equivalent uniform dose (EUD) techniques proposed by JT Lyman (Kutcher et al., 1989).

For heterogeneous irradiation of tissues, the overall TCP is defined as the product of the probability of killing all clonogens in each differential volume element (Vi) of a tumor target irradiated with a dose (Di) in the Poisson statistics model. Furthermore, the overall TCP takes into account of the cell survival fractions assuming the single hit mechanism of the cell damage. It can be expressed as:

$$
\mathrm{TCP}=\left[\frac{1}{2}\right]^{\sum_{\mathrm{i}} \mathrm{V}_{\mathrm{i}} \mathrm{exp}\left[\frac{2 \gamma 50\left(1-\mathrm{Di}_{\mathrm{i}} / \mathrm{TCD} 50\right)}{\ln 2}\right]}
$$

Where the parameters TCD50 and $\gamma 50$ are the dose and normalized slope at $50 \%$ probability of tumor control in the target. HART utilizes the above expression in order to predict the dose response probability of the tumor.

The NTCP indices for normal tissues irradiated during the radiotherapy treatment based on the sigmoid dose-response and the equivalent uniform dose concept is expressed as:

$$
\mathrm{NTCP}=\phi\left[\frac{\mathrm{EUD}-\mathrm{TD50}}{\mathrm{m} \text { TD50 }}\right]
$$

The EUD or the generalized mean dose (GMD) represents the dose that, if delivered uniformly to the normal tissues or the entire critical structure, would produce the same effect as the heterogeneous dose distribution as specified by the DVH. The parameter $\mathrm{m}$ controls the slope of the dose-response curve, and TD50 determines the position of a dose response curve at $50 \%$ chance of complication in the critical structure.
Furthermore, GMD can be expressed as:

$$
\mathrm{GMD}=\left(\sum_{\mathrm{i}} \mathrm{vid}_{\mathrm{i}}^{1 / \mathrm{n}}\right)^{\mathrm{n}}
$$

Where $\mathrm{n}$ determines the dose-volume dependence of a tissue which is deterministic for differ-ences in tissue architecture. The above expressions were implemented into HART to predict the NTCPs for the neighbouring structures of the target in a specific treatment plan (Kutcher et al.,1989). The clinical outcome of the treatment and the toxicity of the OAR like dysphagia and xerostomia were compared with these NTCP values calculated from HART software.

\section{Results}

The plan indices and QFs were analyzed for the IMRT treatment of $20 \mathrm{H}$ and $\mathrm{N}$ patients using HART software with inputs from DVH parameters calculated using Eclipse TPS were tabulated in Table 2, and the mean values of UPIs and QFs plans were determined

Table 1. Patient's Demographics

\begin{tabular}{lc}
\hline Characteristics & Number (Percentage) \\
\hline Total No. of Patients & $20(100)$ \\
Sex & $17(85)$ \\
$\quad$ Male & $3(15)$ \\
Female & \\
Age in years & $5(25)$ \\
$41-50$ & $8(40)$ \\
$51-60$ & $7(35)$ \\
$61-70$ & \\
Primary Site & $14(70)$ \\
Hypopharynx & $6(30)$ \\
Oropharynx & \\
TNM Stage & $3(15)$ \\
Stage III & $14(70)$ \\
Stage IV A & $3(15)$ \\
Stage IV B &
\end{tabular}

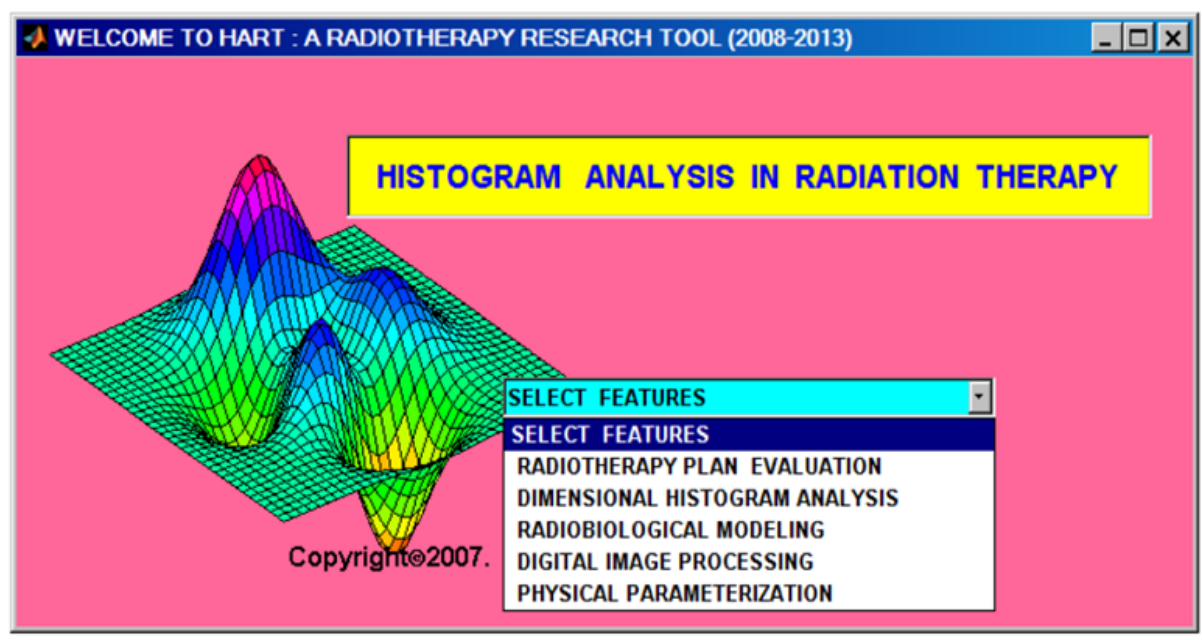

Figure 1. Screen Shot of the GUI of HART Software 


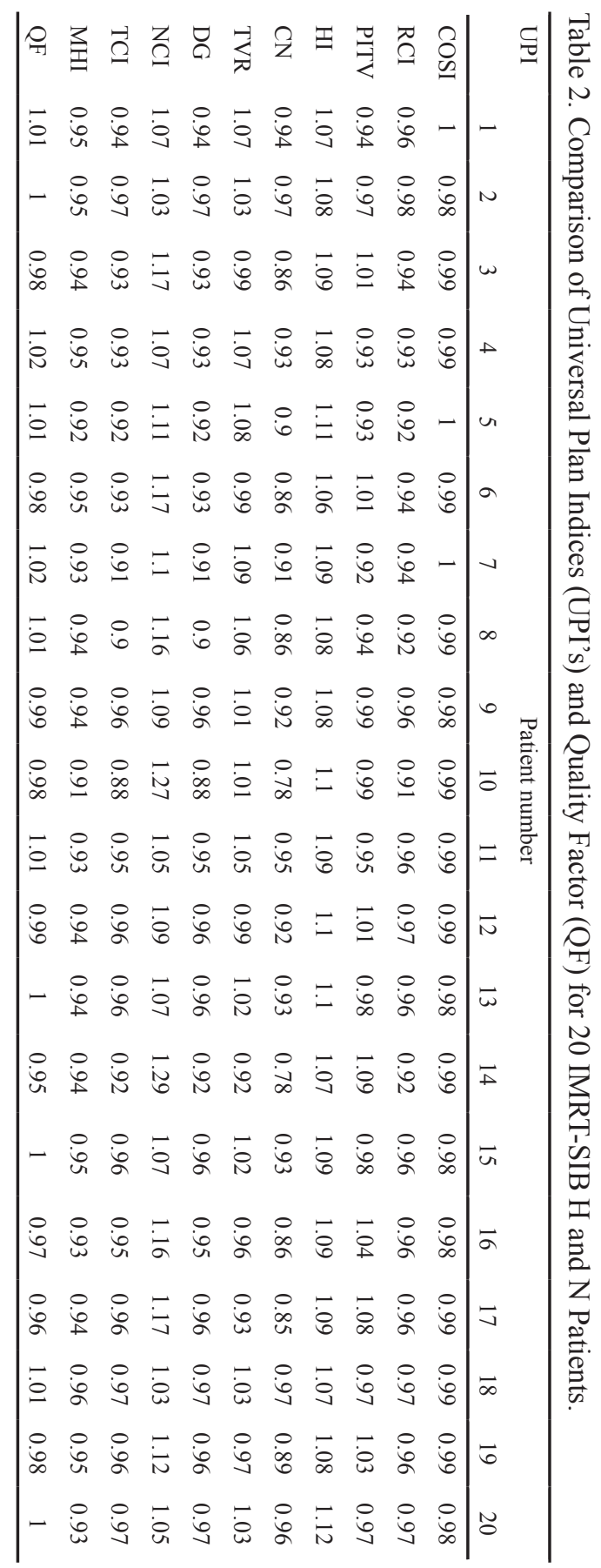

systematically were shown in Figures 2 and 3.

The short term clinical outcomes of the radiotherapy treatments were in terms of Poisson's TCP indices and EUD based JT Lyman NTCP indices with respective acute toxicities. The input parameters were given from cumulative DVH data from TPS, and other values were taken from Luxton et al., (2008). For tumor control TCD50 $=63.8 \mathrm{~Gy}$ and $\alpha / \beta=10$ Gy were used to compute the TCP of the target in the corresponding plans. Similarly, the complications in the normal tissue organs were calculated by using the cell surviving parameters. The cell survival parameters used for bilateral parotid is TD 50,5 $=46$ Gy, for larynx, TD 50,5=70 Gy and for constrictors TD 50,5=50 Gy. The NTCP values of these OARs were used to correlate acute toxicity levels, which led to complications.

The Poisson TCP index and the JT Lyman NTCP indices for all 20 patients are estimated from HART

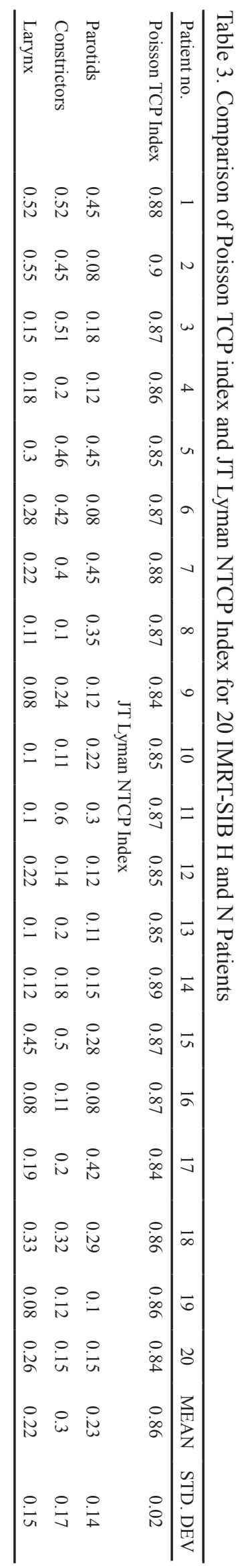

software with an input of DVH parameters is tabulated in Table 3. The mean Poisson TCP index was found to be 0.86 \pm 0.02 and the mean JT Lyman NTCP index for bilateral parotid, constrictors, larynx were found to be $0.23 \pm 0.14$, 


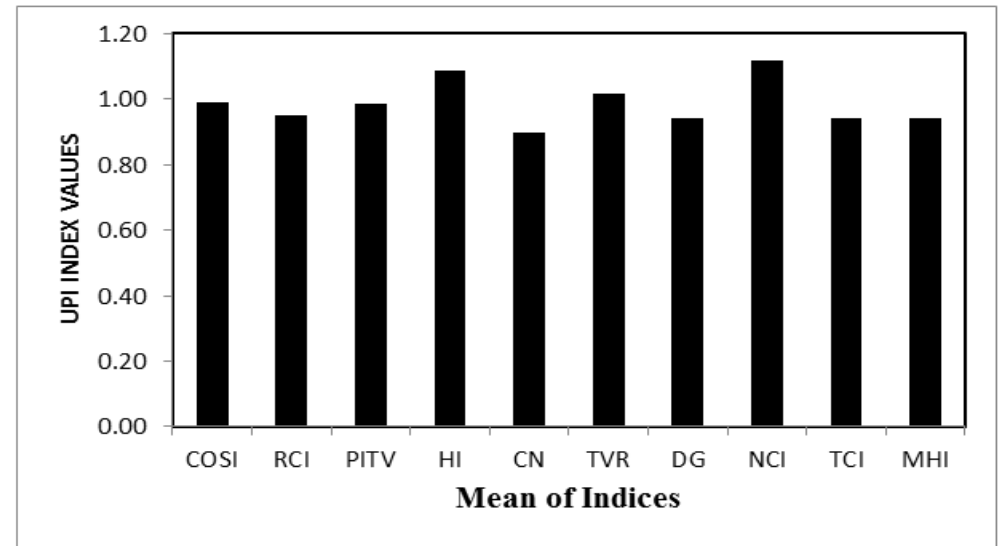

Figure 2. Comparison of Mean UPI Indices for 20 IMRT-SIB H and N Patients

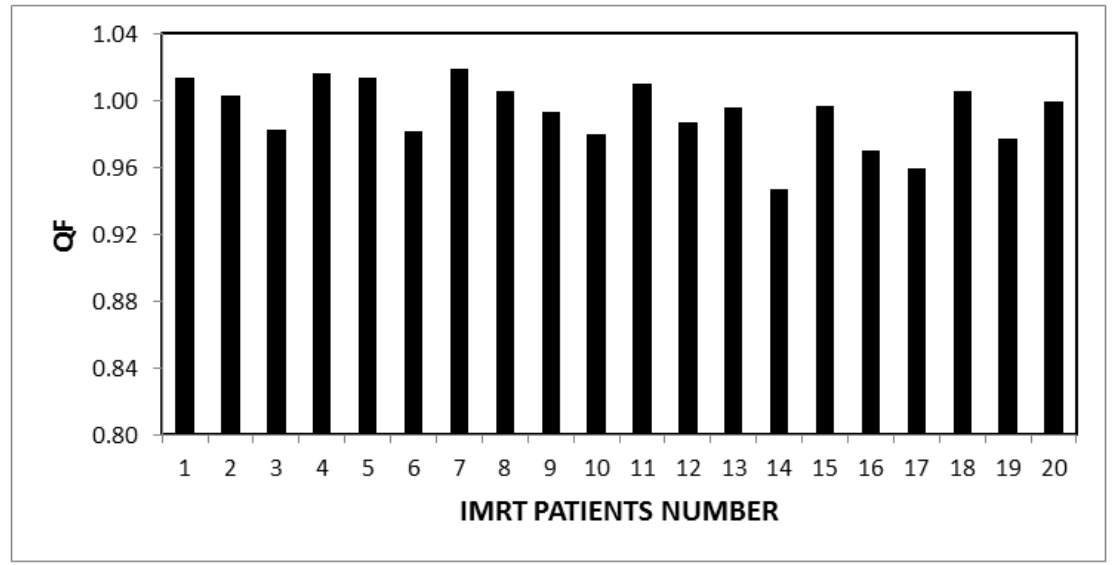

Figure 3. Comparison of Overall QF for 20 IMRT-SIB H and N Patients

$0.30 \pm 0.17$ and $0.22 \pm 0.15$. The correlation between these results and its clinical findings in terms of acute toxicities is described as below.

The overall clinical response was assessed among the group of 20 patients and found that 16 patients $(80 \%)$ were complete response category and four patients (20\%) were in partial response after the post-treatment mean follow up of 3 months. The severity of xerostomia and the corresponding NTCP values of the bilateral parotids is shown in Figure 4. The correlation between them was found to be positive with a coefficient of 0.55 , and it was statistically significant at $\mathrm{P}<0.01$. Figure 5 is shown the severity of dysphagia with NTCP values of constrictors and it shows a moderate correlation with the coefficient of 0.59 , and it was statistically significant at $\mathrm{P}<0.01$. Similar way, the severity of dysphagia with NTCP of Larynx is shown in Figure VI. It was noticed that there is a moderate correlation between the severity with respective NTCP indices with the coefficient of 0.37 , and it was statistically significant at $\mathrm{P}<0.01$.

\section{Discussion}

In HART, the UPI set has been defined by summarizing

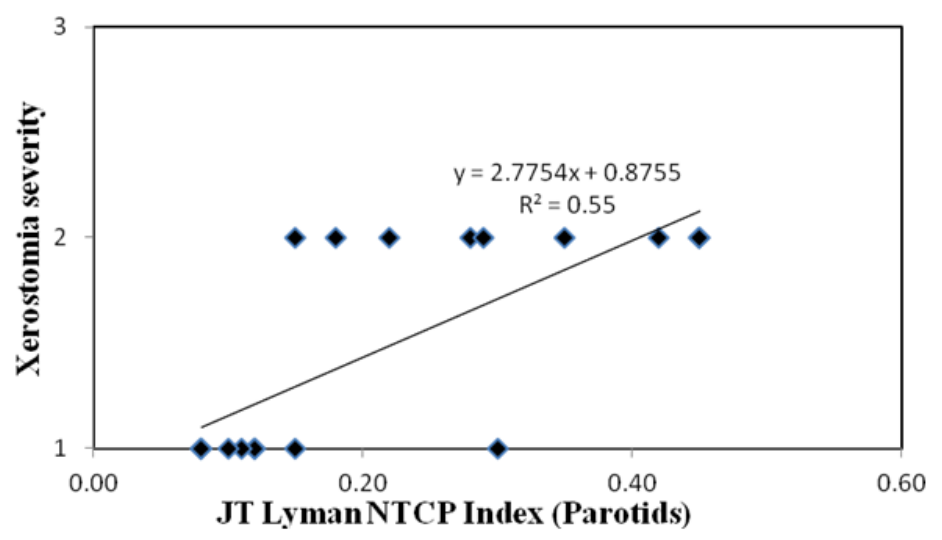

Figure 4. Correlation between the NTCP Indices of the Bilateral Parotids and Its Clinical Findings in Terms of Severity of Xerostomia for 20 IMRT-SIB H and N Patients 


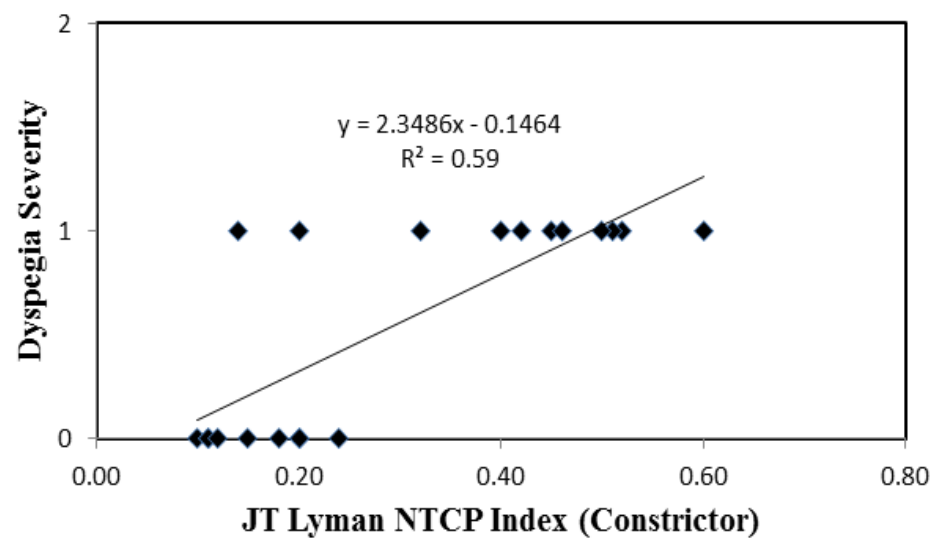

Figure 5. Correlation between the NTCP Indices of the Constrictors and Its Clinical Findings in Terms of Severity of Dyspegia for 20 IMRT-SIB H and N Patients

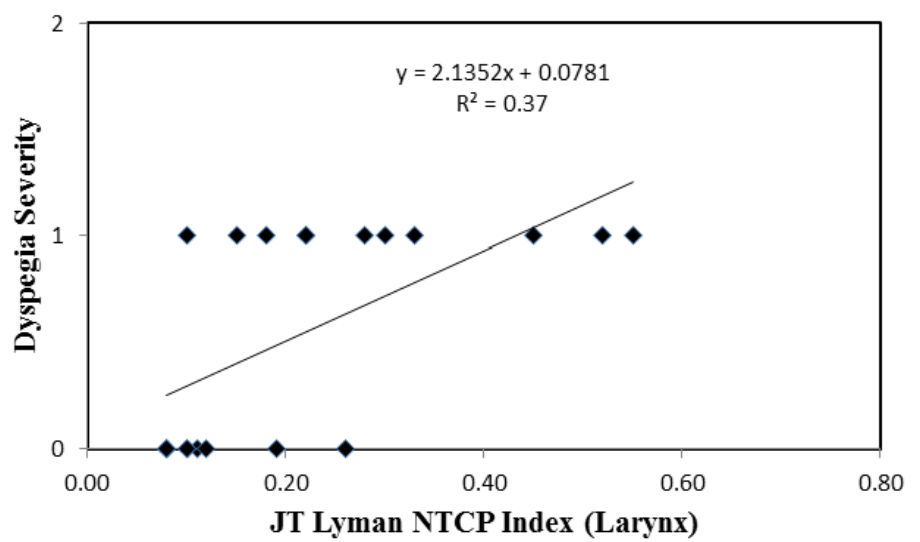

Figure 6. Correlation between the NTCP Indices of the Larynx and Its Clinical Findings in Terms of Severity of Dyspegia for 20 IMRT-SIB H and N Patients

various recognized plan indices for plan evaluation of IMRT treatments and an overall QF of a plan were also determined by combining the relative assessments of all plan indices using DVH data in the UPI set (Pyakuryal et al., 2010). The ideal UPI scores for all plan indices should be unity, and the typical value of QF equals unity represents an ideal plan. The deviation from unity refers to underdose or overdose treatments in the corresponding plan. Our present study result shows that the mean QF of all 20 patients has been calculated as $0.993 \pm 0.02$, which is close to unity.

Recent gains in the management of HandN cancer have been achieved due to concurrent chemo-radiotherapy with altered fractionated IMRT technique (Fang et al., 2008). In a dual institutional study, results of 33 patients with oropharyngeal primaries in the head and neck region indicated a strong correlation between the severities of xerostomia and dysphagia with NTCP of bilateral parotids and oesophagus respectively, but not with the larynx (Narayanasamy et al.,2015). Peponi et al., (2011) have evaluated the objective and subjective long-term swallowing function, and to relate the radiation dose delivered to critical structures of a total 82 patients with stage III/IV of the carcinoma of larynx, oropharynx, or hypopharynx with SIB-IMRT either alone or in combination with chemotherapy. In their results with 32 months mean post treatment follow-up, grade $3 / 4$ objective toxicity was assessed in 10\%. Beetz et al., (2012) have investigated the significance of the radiation dose in salivary glands in a multicenter study and their results with 6 months follow-up data showed that, the mean parotid dose was one of the important predictors of moderate to severe xerostomia. Chao et al., (2001) observed a correlation between parotid mean dose and the fractional reduction of stimulated saliva output at 6 months after the completion of radiation therapy and concluded that sparing of the parotid glands translates into objective and subjective improvement of both xerostomia and QOL scores in patients with head-and-neck cancers receiving radiation therapy.

Dirix et al., (2009) have evaluated the dysphagia after chemo radiotherapy for locally advanced HNSCC and examined its correlation with clinical and dosimetric parameters. In which they have found that, increased dose to a large volume of swallowing structures is reported to result in higher levels of dysphagia. Caglar et al., (2008) evaluated early swallowing after IMRT with or without chemotherapy and attempted to determine the clinical and/or dosimetric factors correlating with swallowing toxicity. They did not find any correlation with the superior constrictors dose and early dysphagia.

In the present study which includes of oropharynx and hypopharynx with accelerated IMRT treatment with concurrent chemotherapy, the severity of xerostomia and 
dysphagia has a moderate correlation with NTCP values of bilateral parotids, constrictors and larynx respectively.

In conclusion, we have analysed twenty patients of HandN cancer patients who underwent accelerated SIBIMRT with concurrent chemotherapy using physical and radiobiological indices in comparison with their clinical outcomes in terms of acute toxicities. The physical assessment using mean quality factors based on UPI were found to be close to unity which correlates to be a better IMRT plan. The respective correlation between the toxicities of the OAR and their NTCP values depends not only on the IMRT plan dose-volume parameters but also on the available follow up data. The present study suggested an existence of the moderate correlation between the calculated NTCP values and their respective sevirities of the OAR's. The accelerated IMRT with concurrent chemotherapy is a clinically feasible option in the treatment of locally advanced HNSCC with encouraging initial tumor response and acceptable acute toxicities.

\section{Acknowledgments}

\section{Conflict of Interest}

None Declared.

\section{Funding Information}

No funding received.

\section{References}

Akpati H, Kim C, Kim B, Park T, Meek A (2008). Unified dosimetry index (UDI): a figure of merit for ranking treatment plans. J Appl Clin Med Phys, 9, 99-108.

Baumann M, Petersen C (2005). TCP and NTCP: A basic introduction. Rays, 30, 99-104.

Beetz I, Schilstra C, Burlage FR, et al (2012). Development of NTCP models for head and neck cancer patients treated with three dimensional conformal radiotherapy for xerostomia and sticky saliva: The role of dosimetric and clinical factors. Radiother Oncol, 105, 8693.

Cadman P, Bassalow R, Sidhu NP, Ibbott G, Nelson A (2002). Dosimetric considerations for validation of a sequential IMRT process with a commercial treatment planning system. Phys Med Biol, 47, 3001-10.

Caglar HB, Tishler RB, Othus M, et al (2008). Dose to larynx predicts for swallowing complications after intensity-modulated radiotherapy. Int J Radiat Oncol Biol Phys, 72, 1110-8.

Chao KS, Deasy JO, Markman J, et al (2001). A prospective study of salivary function sparing in patients with head-and-neck cancers receiving intensity-modulated or three-dimensional radiation therapy: Initial results. Int $J$ Radiat Oncol Biol Phys, 49, 907-16.

Collins SP, Coppa ND, Zhang Y, et al (2006). CyberKnife radiosurgery in the treatment of complex skull base tumors: analysis of treatment planning parameters. Radiat Oncol, $1,46$.

Dirix P, Abbeel S, Vanstraelen B, Hermans R, Nuyts S (2009). Dysphagia after chemoradiotherapy for head and neck squamous cell carcinoma: Dose effect relationships for the swallowing structures. Int J Radiat Oncol Biol Phys, 75, 385-92.

Fang F-M, Chien C-Y, Tsai W-L, et al (2008). Quality of life and survival outcome for patients with nasopharyngeal carcinoma receiving three- dimensional conformal radiotherapy vs. intensity-modulated radiotherapy-A longitudinal study. Int J Radiat Oncol Biol Phys, 72, 356-64.

Kataria T, Sharma K, Subramani V, Karrthick KP, Bisht SS (2012). Homogeneity Index: An objective tool for assessment of conformal radiation treatments. J Med Phys, 37, 207-13.

Knöös T, Kristensen I, Nilsson P (1998). Volumetric and dosimetric evaluation of radiation treatment plans: radiation conformity index. Int J Radiat Oncol Biol Phys, 42, 1169-76.

Kutcher GJ, Burman C (1989). Calculation of complication probability factors for non-uniform normal tissue irradiation: The effective volume method. Int J Radiat Oncol Biol Phys, 16, 1623-30.

Leung L, Chua D, Wu PM (1999). A new tool for dose conformity evaluation of radiosurgery treatment plans. Int $J$ Radiat Oncol Biol Phys, 45, 233-41.

Leung LH, Kan MW, Cheng AC, Wong WK, Yau CC (2007). A new dose volume-based plan quality index for IMRT plan comparison. Radiother Oncol, 85, 407-17.

Luxton G, Keall PJ, King CR (2008). A new formula for normal tissue complication probability (NTCP) as a function of equivalent uniform dose (EUD). Phys Med Biol, 53, 23-36.

Menhel J, Levin D, Alezra D, Symon Z, Pfeffer R (2006). Assessing the quality of conformal treatment planning: a new tool for quantitative comparison. Phys Med Biol, 51, 5363-75.

Narayanasamy G, Pyakuryal AP, Pandit S, et al (2015). Radiobiological evaluation of intensity modulated radiation therapy treatments of patients with head and neck cancer: A dual-institutional study. J Med Phys, 40, 165-9.

Paddick I (2000). A simple scoring ratio to index the conformity of radiosurgical treatment plans. J Neurosurg, 93, 219-22.

Peponi E, Glanzmann C, Willi B, Huber G, Studer G (2011). Dysphagia in head and neck cancer patients following intensity modulated radiotherapy (IMRT). Radiat Oncol, $6,1$.

Pyakuryal A, Myint W K, Gopalakrishnan M, et al (2010). A computational tool for the efficient analysis of dose-volume histograms from radiation therapy treatment plans. J Appl Clin Med Phys, 11, 137- 57.

Warkentin B, Stavrev P, Stavreva N, Field C, Fallone BG (2004). A TCP-NTCP estimation module using DVHs and known radiobiological models and parameter sets. $\mathrm{J} \mathrm{Appl} \mathrm{Clin} \mathrm{Med}$ Phys, 5, 50-63.

Yoon M, Park S, Shin D, et al (2007). A new homogeneity index based on statistical analysis of dose-volume histogram. $J$ Appl Clin Med Phys, 8, 9-17.

This work is licensed under a Creative Commons AttributionNon Commercial 4.0 International License. 